\title{
DETECTION OF BETA-DEFENSINS AND NOS IN HEALTHY AND PATHOLOGICAL NASAL MUCOSA
}

\author{
Hana Pácováa*, Tomáš Kučera ${ }^{\mathrm{b}}$, Jaromír Astl ${ }^{\mathrm{a}}$, Jindřich Martínek
}

${ }^{a}$ Department of Otorhinolaryngology and Head and Neck Surgery, ${ }^{\text {st }}$ Medical Faculty

${ }^{b}$ Institute of Histology and Embryology, ${ }^{\text {st }}$ Medical Faculty, Charles University, Prague, Czech Republic e-mail:Hpac@seznam.cz

Received: September 20, 2004

Key words: Beta-defensin/Nitric oxide synthase/Apoptosis/Cell proliferation/Nasal polyposis

\section{INTRODUCTION}

Endogenously produced anti-microbial peptides beta-defensins and nitric oxide (NO) are important components of the immune response. When this response is disturbed it can have damaging consequences. Nasal polyposis, a common disorder, is an example of such immune dysregulation. In order to establish the possible role of human beta defensin-2 (hBD-2), human beta defensin-3 (hBD-3) and NO in the etiopathogenesis of nasal polyposis, the immunohistochemical detection of hBD-2, hBD-3 and endothelial nitric oxide synthase (eNOS) was performed in healthy and pathological human nasal mucosa. Another aim of this study was to find out whether there is a correlation between the level and localization of these antibacterial agents and the occurrence of cell proliferation and programmed cell death. The apoptotic marker cleaved caspase- 3 and proliferative marker Ki67 were used for the immunohistochemical detection of these processes.

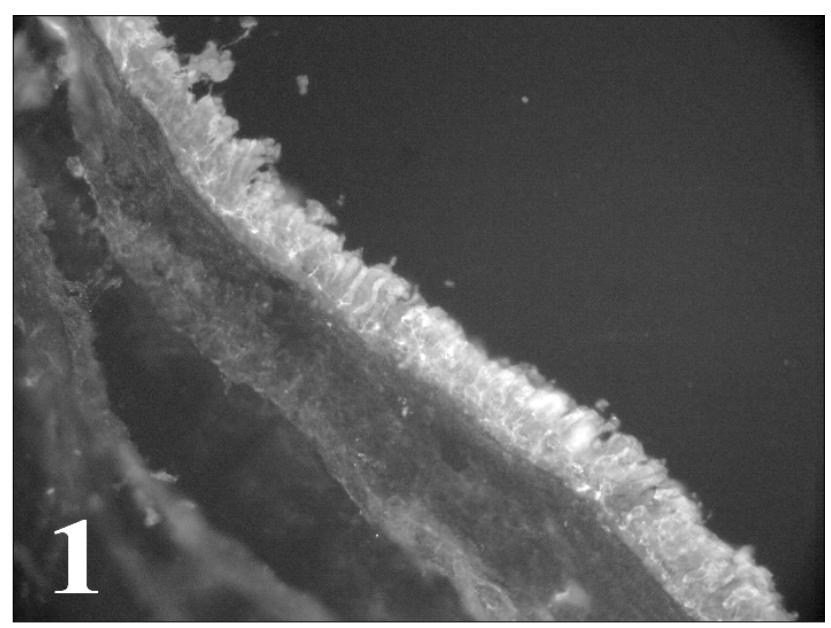

Fig. 1. Immunohistochemical detection of hBD-3 in the surface pseudostratified columnar epithelium of the nasal polyps. Secondary antibody labeled with Cy3. Photograph taken using monochrome camera. Digitally processed. Orig. obj. magn. $40 \mathrm{x}$

\section{METHODS}

The samples of human nasal mucosa from inferior nasal turbinates and nasal polyps from patients with nasal polyposis $(n=9)$ and with the deviation of nasal septum $(n=4)$ were obtained during surgery. Samples were fixed in $4 \%$ paraformaldehyde or snap frozen in liquid nitrogen and kept at $-80^{\circ} \mathrm{C}$ until sectioning. The detection of hBD-3 was carried out on cold acetone-fixed frozen sections by indirect immunofluorescence using rabbit polyclonal antibody and Cy3-labeled secondary antibody. Paraffin sections were used for the detection of hBD-2, endothelial NOS and cleaved caspase-3 with rabbit polyclonal antibodies and Ki-67 with mouse monoclonal antibody via the three-step immunoperoxidase method. Vectastain ABC Elite kit peroxidase and Vector DAB substrate kit were applied for the visualization of biotinylated secondary antibodies. Tissue sections were counterstained with hematoxylin.

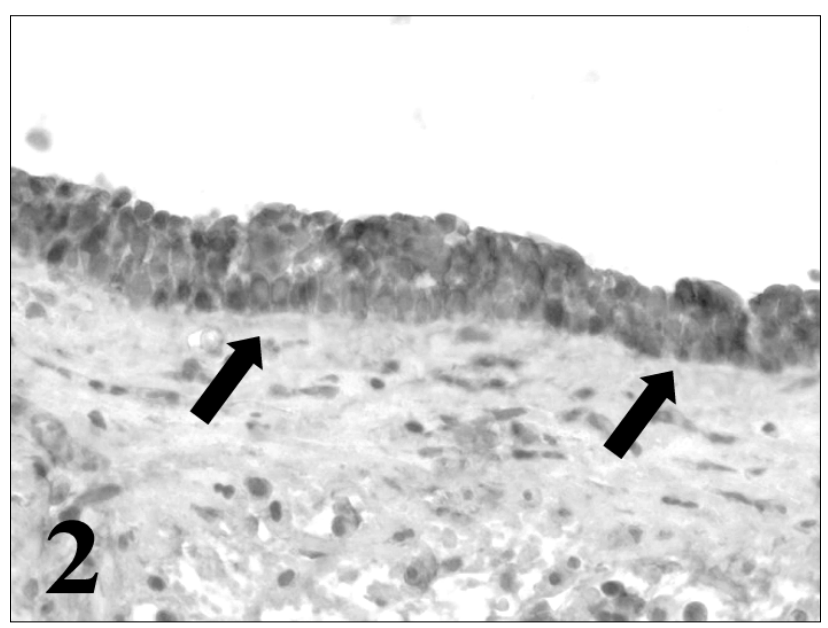

Fig. 2. The result of immunohistochemical detection of hBD-2 in the nasal polyp. The reaction product is localized in the surface pseudostratified epithelium. Immunoperoxidase reaction (DAB). Orig. obj. magn. $63 x$ 


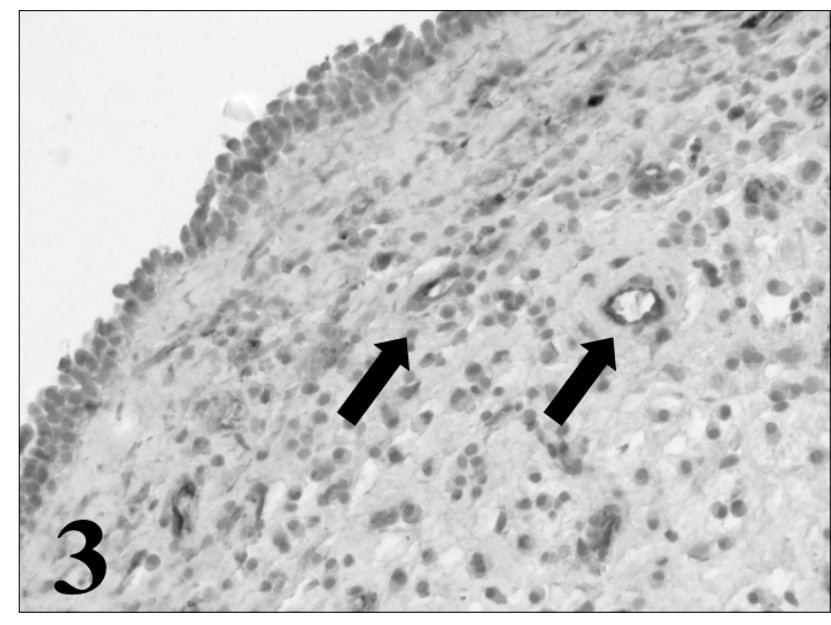

Fig. 3. The result of eNOS detection in the nasal polyp shows the reaction product in the endothelium of vasculature within the lamina propria. Immunoperoxidase reaction (DAB). Orig. obj. magn. $40 \mathrm{x}$

\section{RESULTS AND DISCUSSION}

hBD-3 was localized with variable intensity in the surface epithelial cells and in the epithelial cells of some glands and their ducts in lamina propria of nasal polyps (Fig. 1). Immunopositivity was detected in the cytoplasm, where it had diffuse and in some ductular epithelial cells also a granular pattern. hBD-2 was detected in surface epithelial cells and glandular epithelial cells in the polyps as well as in healthy nasal turbinate (Fig. 2). In some samples it was also present on the surface of ciliated cells. The intensity of immunostaining varied among different samples. NOS-3 was detected in endothelial cells of the vasculature in lamina propria (Fig. 3). The level of immunostaining varied among samples from both polyps and healthy nasal mucosa. The apoptotic marker cleaved caspase-3 was detected only rarely in several examined tissue sections. Immunoreactive apoptotic bodies were occasionally found in the epithelium and some apoptotic cells were present in the connective tissue in lamina propria. The proliferative marker Ki-67 was detected in the nuclei of basal epithelial cells in the nasal mucosa of polyps and also in the epithelium of the inferior nasal turbinate (Fig. 4). In one sample Ki-67 positivity was also found in the capillary endothelium. Ki-67 positive cells in the connective tissue of lamina propria were detected in variable number. There was no significant difference between healthy and pathological tissue.

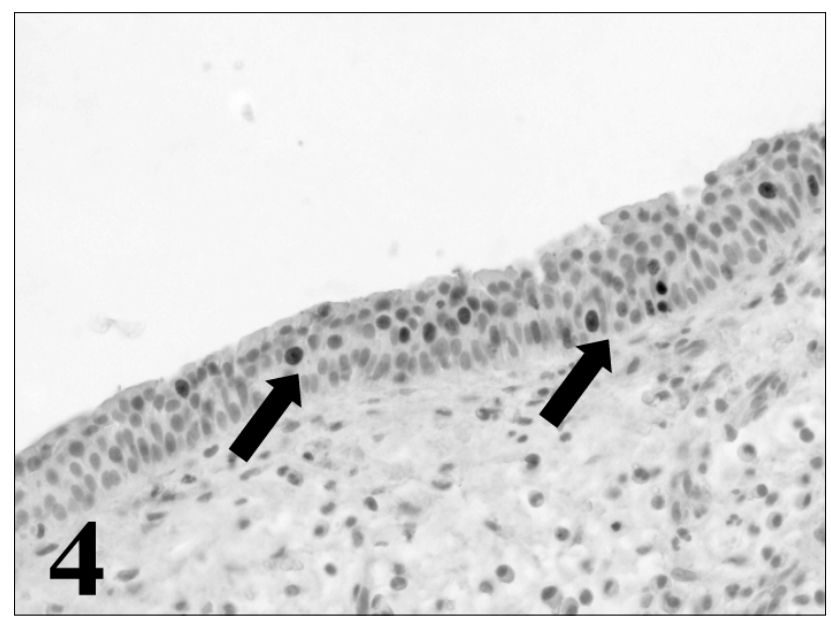

Fig. 4. The proliferation marker Ki-67 labels nuclei of epithelial cells in the basal layer of pseudostratified ciliated columnar epithelium in the nasal polyp. Immunoperoxidase reaction (DAB). Orig. obj. magn. $40 \mathrm{x}$

The preliminary results obtained from immunohistochemical detections of some potential agents that may contribute to the development of nasal polyposis show their relatively widespread expression. Although the production of hBD-2 and hBD-3 are assumed to be induced only in certain inflammatory conditions, it was demonstrated that they could be also expressed in relatively healthy human nasal mucosa. This may indicate that their induction does not require chronic inflammatory stimulation and that they can be expressed as a response to a common bacterial colonization. The NOS-3 is present only in endothelial cells of the vasculature in the lamina propria which does not support its previously reported epithelial localization. Our findings show that the apoptotic process is not a frequent event in nasal mucosa. In addition, no significant difference between the healthy and pathologic tissue was found. On the other hand, numerous proliferating cells were found among epithelial and stromal cells in several samples. The finding of Ki-67 positive endothelium in the inflamed mucosa suggests the stimulation of angiogenesis.

\section{ACKNOWLEDGEMENTS}

This work was supported by the grant FRVŠ No. 986/ 2004 and GAUK 2/2004/C/1.LF. 